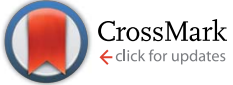

Cite this: RSC Adv., 2015, 5, 29306

Received 27th February 2015

Accepted 16th March 2015

DOI: $10.1039 / \mathrm{c} 5 \mathrm{ra03561a}$

www.rsc.org/advances

\section{Anomalous impact and strain responses in helical carbon nanotube foams $\uparrow$}

\author{
Ramathasan Thevamaran, ${ }^{\text {ab }}$ Mehmet Karakaya, ${ }^{c}$ Eric R. Meshot, ${ }^{\mathrm{d}}$ Andre Fischer, ${ }^{\mathrm{b}}$ \\ Ramakrishna Podila, ${ }^{c}$ Apparao M. Rao ${ }^{c}$ and Chiara Daraio*ab
}

\begin{abstract}
We describe the quasistatic and dynamic response of helical carbon nanotube (HCNT) foams in compression. Similarly to other CNT foams, HCNT foams exhibit preconditioning effects in response to cyclic loading; however, their fundamental deformation mechanisms are unique. In quasistatic compression, HCNT foams exhibit strain localization and collective structural buckling, nucleating at different weak sections throughout their thickness. In dynamic compression, they undergo progressive crushing, governed by the intrinsic density gradient along the thickness of the sample. HCNT microbundles often undergo brittle fracture that originates from nanoscale defects. Regardless of this microstructural damage, bulk HCNT foams exhibit super-compressibility and recover more than $90 \%$ of large compressive strains (up to $80 \%$ ). When subjected to striker impacts, HCNT foams mitigate impact stresses more effectively compared to other CNT foams comprised of non-helical CNTs ( $~ 50 \%$ improvement). The unique mechanical properties we revealed demonstrate that the HCNT foams are ideally suited for applications in packaging, impact protection, and vibration mitigation.
\end{abstract}

\section{Introduction}

Helical carbon nanotubes (HCNTs) have previously been synthesized in several forms, including individual fibers, ${ }^{1}$ selfassembled ropes, ${ }^{2}$ or in macroscopic arrays., ${ }^{3,4}$ Small-scale HCNT fibers have been synthesized for a variety of potential applications such as nano-electronics and nano-mechanical systems, ${ }^{5}$ self-sensing mechanical resonators, ${ }^{6}$ reinforcement in epoxy based composites, ${ }^{7,8}$ and energy applications including fuel cells, hydrogen storage and super-capacitors., ${ }^{\mathbf{9 1 0}}$ Macroscopic arrays of HCNTs have been suggested for applications such as flat panel field emission displays, ${ }^{\mathbf{1 1}}$ electromagnetic shielding, ${ }^{12}$ and energy dissipative cushioning and packaging. ${ }^{13}$ However, unlike non-helical vertically aligned carbon nanotube (VACNT) arrays, ${ }^{\mathbf{1 4 , 1 5}}$ studies on the mechanical response of bulk HCNT foams are sparse in the literature,,$^{7,8,13,16}$ and their

${ }^{a}$ Division of Engineering and Applied Science, California Institute of Technology, Pasadena, CA 91125, USA. E-mail: daraio@ethz.ch

${ }^{b}$ Department of Mechanical and Process Engineering, Swiss Federal Institute of Technology Zurich (ETH Zurich), Zurich 8092, Switzerland

${ }^{c}$ Department of Physics and Astronomy, and Clemson Nanomaterials Center, Clemson University, Clemson, South Carolina 29634, USA

${ }^{d}$ Physical and Life Sciences Directorate, Lawrence Livermore National Laboratory, Livermore, CA 94551, USA

$\uparrow$ Electronic supplementary information (ESI) available: The synchrotron X-ray scattering and mass attenuation experiment methods, SEM images of sample deformation mechanisms, mechanical testing data, and a high-speed microscope video of the dynamic compression of HCNT foam are provided in the Supplementary Information. See DOI: 10.1039/c5ra03561a fundamental deformation mechanisms at different loading rates are not yet fully characterized.

Bulk HCNT foams derive their unique mechanical properties from their hierarchically organized microstructure, characterized by aligned and entangled helical coils of multi-walled CNTs (Fig. 1(a) and (b)). Their mechanical properties are governed by
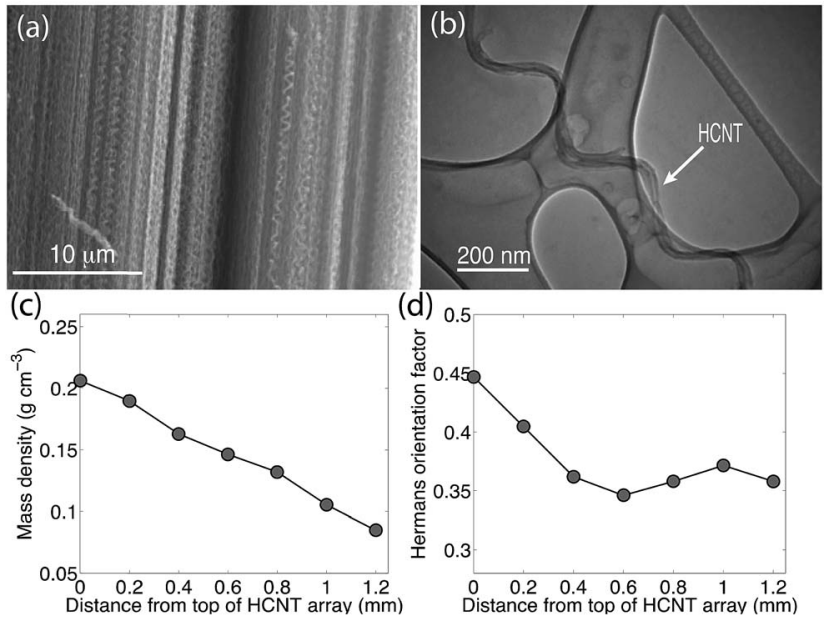

Fig. 1 HCNT foams and structural characteristics: (a) a SEM image of vertically aligned bundles of entangled HCNTs, (b) a TEM image of a representative, individual HCNT, (c) measured mass density gradient along the height of the HCNT foam sample, and (d) measured alignment of the HCNTs within the foam, along the height of the sample. 
the unique behavior of individual nanocoils as well as the collective response of the interacting and entangled neighbors. The carbon nano-coils act like elastic springs, with their deformation behavior governed by geometric nonlinearity. ${ }^{\mathbf{1 7}}$ The spring constant, $k$ of a helical coil is proportional to the quartic power of the diameter, $d$ of the coiled wire (CNT diameter) ( $\left.k \propto d^{4}\right)$, and inversely proportional to the cubic power of the radius, $R$ of the coil $\left(k \propto 1 / R^{3}\right)$. ${ }^{17}$ Such geometric nonlinearity in the deformation of the individual nano-coils leads to an interesting collective mechanical response in the HCNT foams. For example, the contact interaction of a spherical indenter with HCNT foams is highly nonlinear and nonHertzian, and different from the contact interaction of a spherical indenter with VACNT foams. ${ }^{13}$ This highly nonlinear collective response is attributed primarily to the unusual entanglement between neighboring coils and to the collective bending behavior of the coil tips when impacted by a spherical indenter. ${ }^{18}$ The HCNT foams have been shown to mitigate low velocity $\left(0.2 \mathrm{~ms}^{-1}\right)$ impact forces efficiently and fully recover deformation of the order $\sim 5 \mu \mathrm{m}$ (5\% strain). ${ }^{13}$ However, their fundamental deformation mechanisms at large strains and at different strain-rates have not been studied yet.

In this article, we present a comprehensive study of the mechanical response of HCNT foams in both quasistatic and dynamic loading regimes with structural characterizations. We performed structural characterization using synchrotron X-ray scattering and mass attenuation, and correlated the structural characteristics to the observed fundamental deformation mechanisms under compressive loading. We used in situ highspeed microscopy, and ex situ scanning electron microscopy (SEM) and transmission electron microscopy (TEM) to identify the deformation mechanisms that govern the bulk mechanical behavior.

\section{Results and discussions}

\section{Morphology and structure}

The HCNT foams used in this study were synthesized on $\mathrm{Si}$ substrates using a chemical vapor deposition (CVD) process, as described in the experimental methods section. SEM images of the foam's microstructure reveal the uniformity of the coiling and pitch of the HCNTs present in the array (Fig. 1(a)). A TEM image showing a representative nano-coil found in our HCNT foams can be found in Fig. 1(b). The overall thickness of the HCNT foams studied was $\sim 1 \mathrm{~mm}$ and the dominant HCNT diameter and pitch were around $25 \pm 5 \mathrm{~nm}$ and $150 \mathrm{~nm}$, respectively.

We performed small-angle X-ray scattering (SAXS) and mass attenuation measurements using a synchrotron light source to nondestructively quantify the HCNT density and alignment. We used incident photon energy of $10 \mathrm{keV}$ with a beam height of less than $300 \mu \mathrm{m}$ at the sample in order to spatially map structural characteristics along the vertical height of the HCNT foams. Monitoring the drop in X-ray intensity across the sample enabled the determination of the sample's mass density using the Beer-Lambert law. ${ }^{19}$ The mass density was found to decrease linearly with the height of the HCNT foam (with lowest density found on the side of the foam adjacent to the substrate). The foam's average density was $0.15 \mathrm{~g} \mathrm{~cm}^{-3}$ with a $59 \%$ variation along its height (Fig. 1(c)). The HCNT alignment was quantified from the anisotropy of the SAXS patterns, using Herman's orientation factor, $f,^{\mathbf{2 0}, 21}$ where $f$ equals 1 for perfectly aligned CNTs and 0 for random order (no alignment). We found that the alignment decreased from the top to the bottom of the sample, with the bulk samples having an average alignment of 0.38 (Fig. 1(d)). Although the HCNTs are generally aligned in the vertical direction, their coiled nature yields a projected zigzag structure, which results in low Herman's orientation factor. The statistical distribution of orientation angles arising from the helical nano-coils is highlighted in the 2D SAXS patterns (see ESI Fig. S1(b) †). Details of the synchrotron X-ray scattering experimental methods and analysis can be found in ESI S1. $\dagger$

\section{Quasistatic response}

We tested the HCNT foams under quasistatic compression cycles, as described in the methods section. The HCNT foams exhibit a hysteretic, nonlinear stress-strain response (Fig. 2(a)) when subjected to quasistatic compressive loading-unloading cycles, similar to the response reported for other typical foam materials ${ }^{22}$ as well as VACNT foams. ${ }^{14}$ The area enclosed by the hysteresis loop represents the energy dissipated at each compression cycle ( $W_{\text {dissipated }}$ in Fig. 2(b)). The HCNT foams also have the ability to recover large compressive strains of up to $80 \%$. When a HCNT foam is compressed multiple times, the loading path differs from cycle to cycle, a characteristic behavior found in materials with preconditioning effects (Fig. 2(a)). The preconditioning is pronounced in the first three loadingunloading cycles, but the mechanical response stabilizes after the third cycle. A similar preconditioning effect was also reported in VACNT foams and was attributed to microstructural rearrangements of the CNTs during the loading-unloading cycles. ${ }^{14,23}$ In the case of HCNT foams, in addition to the microstructural rearrangements, we also observed permanent microstructural damage and brittle fracture of HCNT bundles in the deformed region (Fig. 2(d)). The unloading modulus, the hysteretic energy dissipation (Fig. 2(b)), and the peak stress (ESI Fig. S2 $\dagger$ ) also decrease rapidly within the first three cycles and remain nearly constant for the later cycles, implying that the mechanical properties of HCNT foams are loading-history dependent. The compressive strength (peak stress at $80 \%$ strain) of the HCNT foams $(22.2 \pm 1.4 \mathrm{MPa})$ and the hysteretic energy dissipation $\left(3.38 \pm 0.32 \mathrm{MJ} \mathrm{m}^{-3}\right)$ are comparable to that of the VACNT foams with similar densities. ${ }^{24}$

When an HCNT foam that was subjected to repeated cyclic loading at a moderate strain was compressed beyond the previous maximum strain $(30 \%)$, the loading path changed from the preconditioned path to the pristine sample's loading path (Fig. 2(c)). This change from preconditioned to pristine response suggests that the strain in the sample is localized and the deformation is not uniform. These regions of strain localization (occurring during the first cycle) are also identifiable in the consecutive cycles (second and later cycles), as indicated on Fig. 2(c). This kind of strain localization was also observed for 

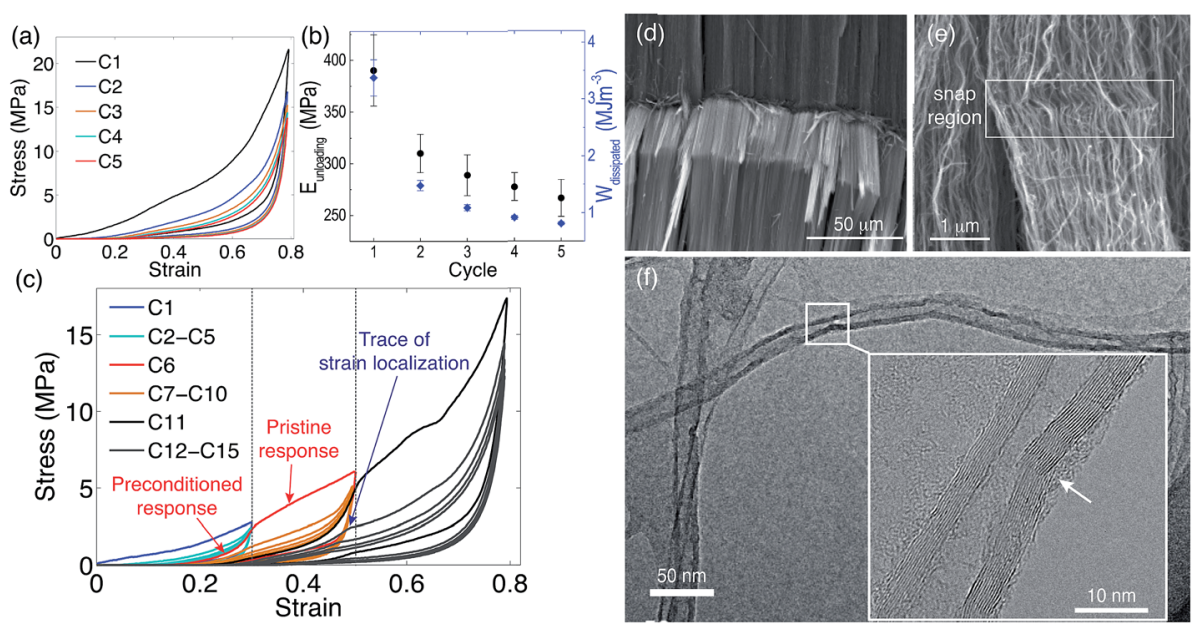

Fig. 2 (a) Stress-strain response of an HCNT foam subjected to five quasistatic compression cycles. (b) Variation of unloading modulus and the hysteretic energy dissipation as a function of the number of loading cycles; error bars represent the standard deviation of three samples measured. (c) Strain localization and loading history dependent response of an HCNT foam. C1-C5, C6-C10 and C11-C15 correspond to compression cycles with $0.3,0.5$, and 0.8 maximum strains, respectively. (d and e) SEM images showing microstructural deformation mechanisms under compression: (d) collective structural buckling of the HCNTs exhibiting brittleness in the response, (e) snap region of a bundle showing that the deformation extends to several pitches of the individual HCNTs, which changes their pristine configuration. (f) TEM images taken at turning points of pristine individual HCNTs revealing defective/broken walls.

VACNT foams, where the vertically aligned bundles of CNTs undergo a well-defined sequential periodic buckling that is governed by the intrinsic density gradient within the material. ${ }^{14,25-27}$ However, the strain localization in HCNT foams is surprising, since previous studies suggested primarily a springlike bulk compressive behavior. ${ }^{13,17}$ We correlate this response to the HCNT foam's microstructure, consisting of long entangled HCNTs with length $(l \sim 1 \mathrm{~mm})$ three orders of magnitude higher than the coil diameter $\left(d_{\text {coil }} \sim 450 \mathrm{~nm}\right) .{ }^{12}$ Due to (i) the very high aspect ratio $\left(l / d_{\text {coil }} \sim 2000\right)$, (ii) the entanglement with neighboring coils, and (iii) the vertical alignment of HCNT bundles, the deformation is localized rather than the whole HCNT foam undergoing a uniform deformation. In situ microscopy and ex situ SEM characterization of a HCNT foam under compression revealed that the strain initially localizes near the substrate where the sample's density is the lowest. After a critical strain of $\sim 10 \%$, localization begins to appear in different regions along the sample's height, without any periodic sequential progression. Several consecutive structural buckles with observable brittleness follow the initial deformation (Fig. 2(d)). An SEM image sequence showing the deformation mechanisms during a quasistatic compression cycle is provided in ESI Fig. S3. $\uparrow$ The SEM images also reveal the presence of several permanent microstructural deformations and HCNT bundles that underwent brittle fracturing during loading. TEM analysis of pristine (as-grown) HCNTs show that the pristine nano-coils inherently have numerous structural defects: the multiwalled HCNTs have highly deformed or defective walls as indicated by arrow in Fig. 2(f). The presence of a large number of such nanoscale defects present in the pristine samples may have led to the fracture of the bundles when compressed. Despite the microstructural damages, the bulk samples show significant recovery upon unloading. This suggests that the interactions among HCNT bundles at the mesoscale play a dominant role in the bulk response of foams, over the nanoscale permanent damages observed in the individual coils.

The presence of quasistatic compression-induced strain localization, at arbitrary regions along the height of the sample, also implies that the influence of the intrinsic density gradient along the thickness of the foam is less significant compared to the influence of the nanoscale defects described above. A closer look at the stress-strain response of the HCNT foams (Fig. 2(c)) shows that the transition regions from preconditioned to pristine loading paths are smooth-in contrast to the sharp transitions observed in VACNT foams. ${ }^{27}$ This implies that the strain localization in HCNT foams is not confined to a narrow region of the foam's thickness (as in the case of the well-defined periodic sequential buckles forming in VACNT foams), ${ }^{\mathbf{1 4 , 2 6}}$ but the deformation extends to several adjacent pitches of the individual HCNTs. This is also evident from SEM images obtained on a compressed sample where several adjacent pitches of the individual helical coils are distorted by bending, buckling and twisting (Fig. 2(e)). An SEM image sequence for a VACNT foam sample subjected to a quasistatic loadingunloading cycle is given in ESI Fig. S4. $\dagger$ Due to these drastically different deformation mechanisms, the loading path of the stress-strain diagram does not show any saw-tooth plateau region with local stress rises and drops, which is a typical characteristic of the formation of localized periodic sequential instabilities. ${ }^{\mathbf{1 4 , 2 6}}$

\section{Dynamic response}

To study the dynamic response of HCNT foams, we performed controlled impact experiments using a flat plunge striker. ${ }^{28}$ In 
the dynamic regime, the HCNT foams exhibit a nonlinear stress-strain response with hysteresis loop (Fig. 3(a) and (b)), similar to the response observed in the quasistatic regime. Fig. 3(a) shows the stress-strain response of an HCNT foam that was impacted repeatedly at increasing velocities. The stressstrain diagrams show the presence of preconditioning effects and strain localization. Similar to the quasistatic response, the preconditioned loading path returns to the pristine loading path as soon as the previous maximum strain is exceeded. In addition to confirming the strain localization in dynamics, this observation suggests that the dynamic loading response is rateindependent. We further verified the rate-independent nature of the loading response of HCNT foams by testing different HCNT foams at controlled impact velocities, between $1 \mathrm{~ms}^{-1}$ and $6 \mathrm{~ms}^{-1}$ (Fig. 3(b)). The stress-strain diagrams followed similar loading paths for the samples tested at increasing velocities (Fig. 3(b)). The dynamic unloading modulus increases with increasing impact velocities, due to the samples reaching higher maximum strains (and densification) with increasing impact velocities (Fig. 3(c)). The dynamic unloading moduli measured were nearly half of the quasistatic unloading moduli (at 0.8 strain), suggesting that HCNT foams are more compliant in dynamic state than quasistatic state. This dynamic effect may
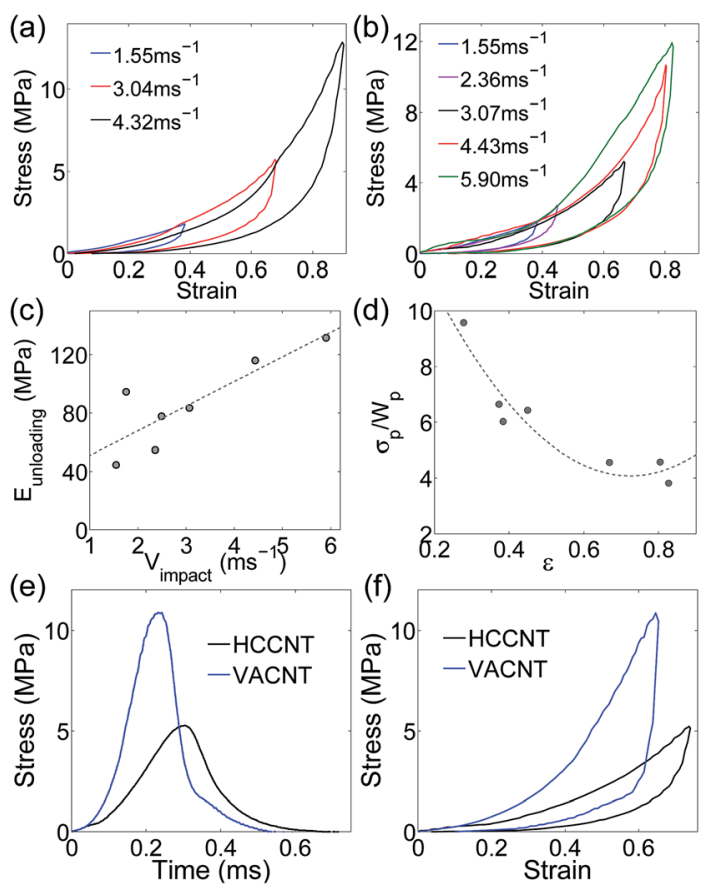

Fig. 3 Impact response of the HCNT foams. (a) Response of an HCNT foam subjected to repeated impacts at increasing velocities. (b) Dynamic stress-strain response of different HCNT foams at increasing impact velocities. (c) Dynamic unloading modulus with the impact velocity. (d) Dynamic cushion factor (peak stress divided by energy absorbed up to peak stress) with maximum strain reached on impacts. (e) Characteristic stress-time history of an HCNT foam compared to a VACNT foam with similar density; both samples were impacted at similar velocities $\left(\sim 3 \mathrm{~ms}^{-1}\right.$ ). (f) Dynamic stress-strain response of the HCNT and VACNT foams, for comparison. have arisen from the faster, spring-like pushback response of HCNT foams during striker impacts.

To show the ability of the HCNT foams to cushion dynamic impacts, we plot the variation of dynamic cushion factor with the maximum strain reached on impact (Fig. 3(d)). The dynamic cushion factor is calculated by dividing the peak stress by the energy absorbed by the sample up to the peak stress. A decrease in peak stress and/or an increase in energy absorption reduce the dynamic cushion factor-characteristics that are beneficial in impact-protective applications. The dynamic cushion factors of HCNT foams are comparable to those of VACNT foams with similar densities. ${ }^{15}$ ESI Fig. S5(a) $\dagger$ presents a comparison of the dynamic cushion factor obtained in HCNT foams and VACNT foams with comparable densities. Even though the HCNT foams and VACNT foams exhibit similar dynamic cushion factors, it should be noted that the VACNT foams exhibit higher hysteretic energy dissipation (ESI Fig. S5(b) $\dagger$ ), by reaching higher peak stresses for a given impact velocity. HCNT foams, however, perform better in damping the impact stress amplitude in the considered range of impact velocities (ESI Fig. S5(c) $\dagger$ ). This improved damping is also evident from the comparison of characteristic dynamic stress-time histories (Fig. 3(e)), and dynamic stress-strain diagrams (Fig. 3(f)), for HCNT foams and VACNT foams impacted at similar velocities $\left(2.99 \pm 0.07 \mathrm{~ms}^{-1}\right)$. At this impact velocity $\left(2.99 \pm 0.07 \mathrm{~ms}^{-1}\right)$, the HCNT foams show 53\% improved impact stress damping over the VACNT foams. The HCNT foams deform more at moderate stress levels and the stress profiles span over a longer duration compared to VACNT foams. This demonstrates that HCNT foams mitigate impacts more effectively by reducing the amplitude of transmitted stresses in the considered range of impact velocities.

The specific damping capacity-i.e., the hysteretic energy dissipated normalized by the energy absorbed up to the peak stress - of all the HCNT foams tested in this study is on average $\sim 0.56 \pm 0.07$. This implies that $\sim 45 \%$ of the energy absorbed by the HCNT foams is stored elastically and released as the striker gains rebound velocity. VACNT foams with similar densities stored only $28 \%$ of the absorbed energy as elastic energy and dissipated the rest (72\%) through hysteresis ${ }^{\mathbf{1 5}}$ (see also ESI Fig. S5(b) $\dagger$ ). The higher elasticity of the HCNT foams is also observable from their narrower hysteresis compared to the VACNT foams. This comparison demonstrates the fundamental role of the helically coiled microstructure of the HCNT foams as opposed to the straight CNT structure of the VACNT foams.

We characterized the fundamental deformation mechanisms during impact using in situ high-speed microscopy. ${ }^{28}$ Characteristic deformation micrographs and the corresponding dynamic stress-strain diagram of an HCNT foam impacted at $4.43 \mathrm{~ms}^{-1}$ are shown in Fig. 4 and in ESI† Video. As evident from the image sequence, when the HCNT foam is impacted it undergoes an initial compression without apparent deformation localization. Then, crushing initiates in the low-density region of the sample adjacent to the substrate and progresses as the striker compresses the foam. After reaching the peak stress at maximum compression (image 4 of Fig. 4), the sample unloads rapidly by pushing the striker back and eventually 


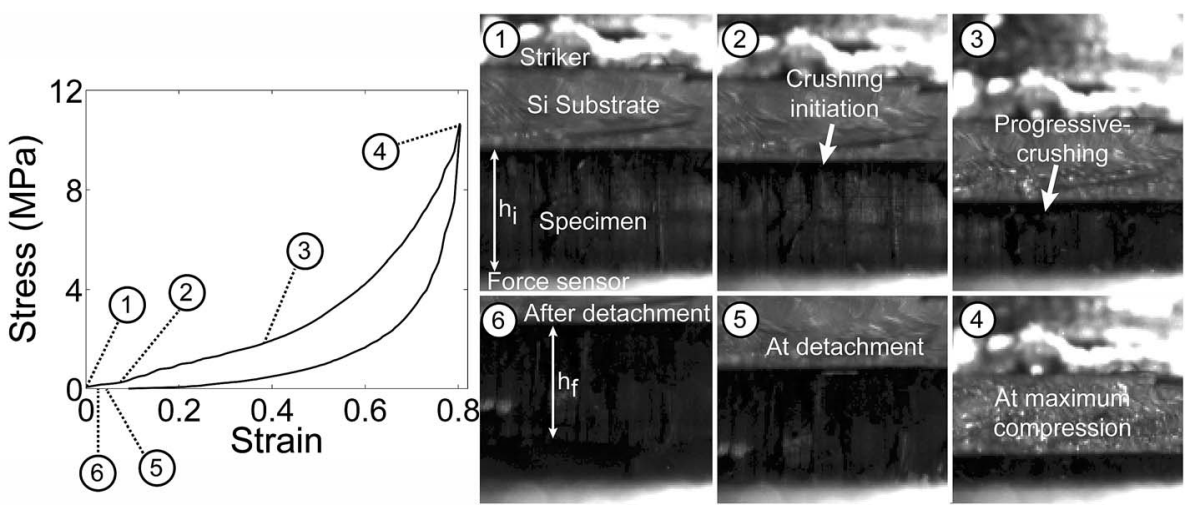

Fig. 4 Stress-strain response and deformation micrographs of an HCNT foam impacted by a striker at $4.43 \mathrm{~ms}^{-1}$. In the dynamic stress-strain diagram (left figure) the circled numbers identify snapshots (shown on the right) selected from the high-speed microscopic image sequence.

detaches from the force sensor. This deformation mechanism in dynamic loading is significantly different from the previously described quasistatic deformation mechanisms of HCNT foams: the intrinsic density gradient governs the progressive deformation in the dynamic state whereas, in the quasistatic compression state, the presence of nanoscale defects dominates the strain localization at arbitrary weak locations. At high impact velocities, the edges of the samples underwent brittle fracture (image 5 of Fig. 4) and a plume of fractured debris could be seen flying off the sample on the high-speed video, when the sample detaches from the force sensor [ESI $\dagger$ Video]. Despite the presence of these microscale fractures, the HCNT foam shown in Fig. 4 recovered $90 \%$ of its bulk compressive strain upon unloading. All HCNT foams tested in impact showed a significant bulk recovery, on average $91.5 \pm 6.3 \%$.

\section{Conclusions}

We studied the mechanical response of HCNT foams subjected to quasistatic and dynamic loadings and identified their fundamental deformation responses. In the quasistatic regime, HCNT foams present strain localizations and structural buckles occurring at arbitrary, weak sections through the sample thickness. Micro-scale brittle fracture of HCNT bundles is also commonly observed, although in bulk, all samples recover most of their deformation. We supported the mechanical tests with SEM/TEM analysis and identified the microstructure contribution to the observed deformation mechanisms and the bulk recovery of the sample. In the dynamic regime, the HCNT foams follow different deformation mechanisms, characterized by the progressive crushing. We correlate this progressive crushing to the intrinsic density gradient that we quantitatively measured using synchrotron X-ray scattering and mass attenuation. We compared the response of HCNT foams to VACNT foams and identified significantly different rate-dependent micro-scale deformation mechanisms. HCNT foams also exhibit better impact absorption characteristics compared to VACNT foams in the considered range of impact velocities. These observations suggest that the HCNT foams can serve as excellent candidates in developing advanced protective materials for energy dissipation and impact absorption.

\section{Experimental methods}

\section{Synthesis of HCNT foams}

HCNT foams were synthesized using a two-stage thermal chemical vapor deposition (CVD) process. The two stages in our CVD reactor - the preheater and the furnace - were maintained at 200 and $700{ }^{\circ} \mathrm{C}$, respectively. The silicon wafers $(3 \mathrm{~cm} \times 3 \mathrm{~cm})$ were then placed in a CVD chamber and heated from room temperature to reaction temperature in the presence of an argon (Ar) flow rate of $500 \mathrm{sccm}$ (cubic centimeter per minute at standard temperature and pressure) and $\mathrm{H}_{2}$ flow rate of 100 sccm. The rate of temperature increase was $15^{\circ} \mathrm{C} \mathrm{min}{ }^{-1}$ during heating. Ferrocene and xylene were used as the catalyst precursor and carbon source, respectively. The In and Sn sources (indium isopropoxide and tin isopropoxide) were dissolved in a xylene-ferrocene mixture where the ratio of $\mathrm{C}: \mathrm{Fe}: \mathrm{In}:$ Sn was maintained at $99.16: 0.36: 0.1: 0.38$, which was then continuously injected into the quartz tube CVD reactor using syringe pump at injection rate of $1.5 \mathrm{ml} \mathrm{h}^{-1}$ after reaching the desired temperature. Simultaneously, acetylene along with Ar carrier gas was passed into the CVD reactor at atmospheric pressure with flow rates $50 \mathrm{sccm}$ and $500 \mathrm{sccm}$, respectively. Subsequently, after $c a .1$ hour of reaction time, the syringe pump and acetylene injection were shut off and the CVD reactor was allowed to cool to room temperature under flowing $\mathrm{Ar}$ atmosphere. The HCNTs were grown on silicon wafer substrates that were placed inside the furnace tube prior to the synthesis run. The resultant HCNT foams were $\sim 1 \mathrm{~mm}$ in height.

\section{Mechanical characterizations}

The quasistatic compression tests were performed on an Instron ElectroPulse E3000 testing system. All the quasistatic experiments were performed at $0.01 \mathrm{~s}^{-1}$ strain rate. The dynamic experiments were performed on an impact testing setup developed in our laboratory. ${ }^{28}$ The experimental setup delivers flat plunge striker impacts on the test specimens at controlled velocities. Geometric moiré transducer was used for microscale 
dynamic displacement measurements while a PCB Piezotronic impact force sensor measured the impact force during impacts. A high-speed microscope was used for in situ visualization and characterization of the micro-scale deformation mechanisms.

\section{TEM sample preparation}

TEM samples were prepared by dispersing HCNT arrays in a $2 \%$ sodium dodecyl sulfate (SDS) solution ${ }^{29}$ using tip sonication. A drop of the HCNT suspension was transferred to a holey carbon grid for the transmission electron microscopy.

\section{Acknowledgements}

We acknowledge Fabian Gramm (ScopeM, ETH Zurich) for assistance in TEM imaging and Jan Rys (ETH Zurich) for assistance in SEM imaging. We acknowledge financial support from the Institute for Collaborative Biotechnologies (ICB) under the contract W911NF-09-D-0001 with the Army Research Office (ARO). A portion of this work was performed under the auspices of the U.S. Department of Energy by Lawrence Livermore National Laboratory under Contract DE-AC52-07NA27344. X-ray characterization was performed at beamline 7.3.3 at the Advanced Light Source, which is supported by the Director, Office of Science, and Office of Basic Energy Sciences, of the U.S. Department of Energy under Contract no. DE-AC02-05CH11231.

\section{References}

1 X. B. Zhang, X. F. Zhang, D. Bernaerts, G. van Tendeloo, S. Amelinckx, J. van Landuyt, V. Ivanov, J. B. Nagy, P. Lambin and A. A. Lucas, Europhys. Lett., 1994, 27, 141.

2 C.-J. Su, D. W. Hwang, S.-H. Lin, B.-Y. Jin and L.-P. Hwang, PhysChemComm, 2002, 5, 34-36.

3 V. Bajpai, L. Dai and T. Ohashi, J. Am. Chem. Soc., 2004, 126, 5070-5071.

4 W. Wang, K. Yang, J. Gaillard, P. R. Bandaru and A. M. Rao, Adv. Mater., 2008, 20, 179-182.

5 T. Hayashida, L. Pan and Y. Nakayama, Phys. B, 2002, 323, 352-353.

6 A. Volodin, D. Buntinx and M. Ahlskog, Nano Lett., 2004, 4, 1775-1779.

7 X. Li, K. Lau and Y. Yin, Compos. Sci. Technol., 2008, 68, 2876-2881.
8 K. Lau, M. Lu and K. Liao, Composites, Part A, 2006, 37, 18371840.

9 A. Leela Mohana Reddy, R. I. Jafri, N. Jha, S. Ramaprabhu and P. M. Ajayan, J. Mater. Chem., 2011, 21, 16103-16107.

10 R. B. Rakhi, W. Chen and H. N. Alshareef, J. Mater. Chem., 2012, 22, 5177-5183.

11 N. Yoshikazu, P. Lujun, H. Taichi and Z. Mei, Jpn. J. Appl. Phys., 2001, 40, L235.

12 S. H. Park, P. Theilmann, K. Yang, A. M. Rao and P. R. Bandaru, Appl. Phys. Lett., 2010, 96, 043115.

13 C. Daraio, V. F. Nesterenko, S. Jin, W. Wang and A. M. Rao, J. Appl. Phys., 2006, 100, 064309.

14 A. Cao, P. L. Dickrell, W. G. Sawyer, M. N. Ghasemi-Nejhad and P. M. Ajayan, Science, 2005, 310, 1307-1310.

15 R. Thevamaran, E. R. Meshot and C. Daraio, Carbon, 2015, 84, 390-398.

16 K. T. Lau, M. Lu and D. Hui, Composites, Part B, 2006, 37, 437-448.

17 X. Chen, S. Zhang, D. A. Dikin, W. Ding, R. S. Ruoff, L. Pan and Y. Nakayama, Nano Lett., 2003, 3, 1299-1304.

18 V. Coluci, A. Fonseca, D. Galvão and C. Daraio, Phys. Rev. Lett., 2008, 100, 086807.

19 M. Bedewy, E. R. Meshot, M. J. Reinker and A. J. Hart, ACS Nano, 2011, 5, 8974-8989.

20 E. Meshot and A. Hart, Appl. Phys. Lett., 2008, 113107, 90-93. 21 B. Wang and R. Bennett, J. Phys. Chem. C, 2007, 5859-5865.

22 L. Gibson and M. Ashby, Cellular solids, Cambridge University Press, 2nd edn, 1999.

23 J. Suhr, P. Victor, L. Ci, S. Sreekala, X. Zhang, O. Nalamasu and P. M. Ajayan, Nat. Nanotechnol., 2007, 2, 417-421.

24 J. R. Raney, A. Misra and C. Daraio, Carbon, 2011, 49, 36313638.

25 O. Yaglioglu, MIT thesis, Massachusetts Institute of Technology, Cambridge, MA, USA, 2007.

26 S. B. Hutchens, L. J. Hall and J. R. Greer, Adv. Funct. Mater., 2010, 20, 2338-2346.

27 J. Raney, F. Fraternali and C. Daraio, Nanotechnology, 2013, 24, 255707.

28 R. Thevamaran and C. Daraio, Exp. Mech., 2014, 54, 13191328.

29 R. Di Giacomo, B. Maresca, A. Porta, P. Sabatino, G. Carapella and H. Neitzert, IEEE Trans. Nanotechnol., 2013, 12, 111-114. 